\title{
Using global positioning system methods to explore mobility patterns and exposure to high HIV prevalence neighbourhoods among transgender women in New York City
}

\author{
William C. Goedel, ${ }^{1}$ Seann D. Regan, ${ }^{2}$ Basile Chaix, ${ }^{3}$ Asa Radix, ${ }^{4}$ Sari L. Reisner, ${ }^{5-7}$ \\ Aron C. Janssen, ${ }^{8}$ Dustin T. Duncan ${ }^{2}$ \\ ${ }^{1}$ Department of Epidemiology, Brown University School of Public Health, Providence, RI, USA; ${ }^{2}$ Social and \\ Spatial Epidemiology Unit, Department of Epidemiology, Columbia University Mailman School of Public Health, \\ New York, NY, USA; "Pierre-Louis" Institute of Epidemiology and Public Health, "Pierre et Marie Curie" \\ University, Paris, France; ${ }^{4}$ Callen-Lorde Community Health Center, New York, NY, USA; ${ }^{5}$ Division of General \\ Pediatrics, Boston Children's Hospital, Harvard Medical School, Boston, MA, USA; ${ }^{6}$ Department of \\ Epidemiology, Harvard T.H. Chan School of Public Health, Boston, MA, USA; ${ }^{7}$ The Fenway Institute, Fenway \\ Health, Boston, MA, USA; ${ }^{8}$ Department of Child and Adolescent Psychiatry, School of Medicine, New York \\ University, New York, NY, USA
}

\begin{abstract}
The aim of this study was to assess mobility patterns among a sample of transgender women $(\mathrm{n}=14)$ in New York City via survey and Global Positioning System (GPS) monitoring. We found varying levels of concordance between the residential neighbourhood and each of the non-residential contexts: $64.3 \%$ considered the neighbourhood that they socialised in most often to be different
\end{abstract}

Correspondence: Dustin T. Duncan, Columbia University Mailman School of Public Health, Department of Epidemiology, Social and Spatial Epidemiology Unit, 766 West 168th Street, Room 715, New York, NY 10032, USA.

E-mail: dd3018@columbia.edu

Key words: Spatial epidemiology; Transgender; HIV; Mobility; Global positioning system.

Conflict of interest: the authors declare no potential conflict of interest.

Funding: this work was funded by a grant from the New York University College of Arts and Science Dean's Undergraduate Research Fund (Principal Investigator: William C. Goedel, BA). At the time of analysis and writing, Dustin T. Duncan, ScD was supported in part by grants from the National Institutes of Health (R01MD013554, R01MH112406, and R03DA039748), and the Centers for Disease Control and Prevention (U01PS005122).

Received for publication: 6 November 2018.

Revision received: 5 April 2019.

Accepted for publication: 10 April 2019

${ }^{\circ}$ Copyright: the Author(s), 2019

Licensee PAGEPress, Italy

Geospatial Health 2019; 14:752

doi:10.4081/gh.2019.752

This article is distributed under the terms of the Creative Commons Attribution Noncommercial License (CC BY-NC 4.0) which permits any noncommercial use, distribution, and reproduction in any medium, provided the original author(s) and source are credited. from their residential neighbourhood. While participants' residences represented 10 zone improvement plan code tabulation areas (ZCTAs), GPS data were recorded in 124 of 263 ZCTAs $(47.1 \%)$. Overall, 58.2\% $(n=373,262)$ were recorded in ZCTAs in the highest quartile of human immunodeficiency virus (HIV) prevalence. The association between place, community HIV prevalence, mobility, and factors that increase the vulnerability of transgender women to HIV infection are worthy of future investigation in reducing the burden of the HIV epidemic in these communities.

\section{Introduction}

A large body of research has accumulated evidence that neighbourhood contexts and their characteristics influence patterns of health and disease at the population level (Duncan and Kawachi, 2018). However, many of these studies use crude neighbourhood definitions, such as those defined for administrative purposes (e.g., zone improvement plan [ZIP] codes, census tracts) (Duncan et al., 2018). These definitions are most often used to approximate an individual's residential environment and are the most common method for delineating neighbourhood boundaries in the literature on neighbourhood effects on health (Duncan et al., 2018). The use of these boundaries, however, can introduce spatial misclassification, or the errors associated with the incorrect characterisation of a neighbourhood-level feature based on the neighbourhood definition used, as this approach makes isotropic assumptions about both the distribution of characteristics within these units and about an individual's mobility within them (Duncan et al., 2013, 2017).

The geographic scope of everyday life activities is not limited solely to the residential neighbourhood (Næss, 2006). However, the majority of studies have assessed associations between the characteristics of residential neighbourhood environments with health behaviours and outcomes. This focus has been referred to as the residential trap, as these studies exclusively rely on assessment of the local residential neighbourhood and systematically neglect to account for the features of non-residential environments (Chaix, 2009). In the sociological literature on place, spatial polygamy has been defined as the simultaneous belonging or exposure to multiple nested and non-nested, social and graphic, 
real, virtual, and fictional contexts (Matthews, 2011). This concept argues for a comprehensive assessment of all residential and nonresidential contexts visited as well as the spaces in between them and their influence on health behaviours and outcomes. Global positioning system (GPS) methods are perhaps the best available method to accomplish this aim as they record an individual's location continuously over time, capturing all spaces visited with a high level of precision (Chaix et al., 2013). The implementation of GPS methods for this purpose has been shown to be acceptable and feasible among many populations, including men who have sex with men (MSM) and transgender women (TGW) (Duncan et al., 2016a; Goedel et al., 2017), two marginalised populations that are impacted by a wide range of health disparities (Institute of Medicine, 2011).

TGW represent a diverse population of individuals who were assigned male sex at birth and identify with a feminine gender identity (Mayer et al., 2008). Transgender individuals face stigma and discrimination from various sectors within society (Grant et al., 2011), thereby limiting their access to housing (Spicer et al., 2010), healthcare (Cruz, 2014), and public accommodation (Reisner et al., 2015), among other spaces. In addition, TGW are also often subject to aggressive police surveillance due to perceived involvement in sex work (Edelman, 2011). While no studies have been conducted on mobility patterns among TGW, we suspect that mobility patterns among TGW may exhibit varying levels of spatial entrapment (England, 1993), resulting in high levels of concordance between the neighbourhood where an individual resides and the other neighbourhoods where they might work, attend school, or socialise. On the contrary, high rates of housing instability and homelessness (Spicer et al., 2010) may also make this population more mobile than the general population, resulting in lower levels of concordance.

Nonetheless, an examination of mobility patterns and exposure to neighbourhood-level factors may help researchers, practitioners, and policymakers plan future studies of neighbourhood effects on health among this population and determine the most appropriate locations for targeted intervention. Therefore, this study aimed to assess the amount of concordance between various types of neighbourhoods and describe mobility patterns in neighbourhoods with high human immunodeficiency virus (HIV) prevalence among a sample of TGW in New York City (NYC).

\section{Materials and Methods}

\section{Sample recruitment and study design}

Data come from the New York City Transgender Women's Neighborhoods and Health Study a pilot project assessing the acceptability and feasibility of using GPS methods to measure mobility among a sample of $14 \mathrm{TGW}$ in NYC. Recruitment methods and the main aims of the project have been described previously (Goedel et al., 2017). Participants were recruited through community-based methods and asked to complete a survey regarding their demographic and behavioural characteristics, carry a small GPS unit on their person for one week, and return to the study offices to complete a second survey on their perceptions of and experiences with the GPS unit. Potential participants were considered eligible if they were between 18 and 40 years old; reported being assigned male sex at birth; reported their gender identity as female, transgender female, male-to-female transgender, transgen- der woman, or woman or transgender experience (among any one of many diverse feminine gender identities); reported residence in the NYC metropolitan area; reported no plans to leave the NYC metropolitan area during the GPS protocol period; reported no limitations to regular physical activity; reported a willingness to wear a small GPS device for one week; and be able to provide written informed consent to participate. All participants provided written informed consent prior to participation. All protocols were approved by the New York University School of Medicine Institutional Review Board.

\section{Data collection via computer-assisted self-interview}

At their first office visit, participants were asked several questions about commonly visited neighbourhoods. Participants were first asked to select one of five boroughs to indicate their current residence. After selecting a borough, participants were asked to select a named neighbourhood corresponding to their current residence. The response options for named neighbourhoods were based on the published list of named neighbourhood tabulation areas (NTAs) designated by the NYC Office of City Planning. Participants were then asked if the neighbourhoods that they most often worked, socialised in, or accessed healthcare services were different from the neighbourhood they lived in. If the participant provided an affirmative answer, they were then asked to select a borough and named neighbourhood corresponding to this location. For these analyses, we defined mobility in terms of the level of concordance between each of the five neighbourhood types (residence and those visited for work, school, social activities, and healthcare services).

Participants reported on a number of demographic covariates, including age, race, ethnicity, relationship status, current housing status, employment status, educational attainment, annual income, health insurance status, and HIV status. Item text and their response options have been provided previously (Goedel et al., 2017).

\section{Data collection via global positioning system}

Details regarding the GPS protocol have been provided previously (Goedel et al., 2017). Participants were given a QStarz BTQ1000XT unit (QStarz International Ltd., Taipei, Taiwan) to carry on their person for 7 days. The GPS unit was set to record location in 10 -second intervals. Information related to the cleaning and processing of GPS data files for each participant has been previously described (Goedel et al., 2017). Participants provided their current home addresses at their first office visits prior to receiving the GPS unit. All spatial analyses were conducted in ArcGIS (Version 10.4.1, Esri, Inc., Redlands, California).

The space within which a person moves during the course of their day-to-day activities (hereby referred to as one's activity space) (Golledge and Stimson, 1997) has been defined using GPS data with various methods (Perchoux et al., 2013), with each approach making different assumptions about mobility and drawing different boundaries around GPS points. In the current study, the daily path area approach was used. This method, commonly used in behavioural geography research to understand where individuals spend the majority of their time (Kwan, 1999), creates a buffered zone around all GPS points. As done in previous studies (Duncan et al., 2017), a 200-meter buffer was used to create activity space size measurements for each participant. The areas covered by these buffers were calculated in square kilometres and are meant to provide an overall metric for mobility, by measuring the 
total geographic span visited by each participant over the span of a typical week.

As a measurement of the diversity of spatial contexts visited by participants over the span of a typical week, we calculated a count of geographic units with GPS data points contained within them (for example, a participant may have had GPS data points recorded within two unique ZIP codes). To capture this diversity of spaces at varying scales, several geographic units used in these analyses, including census tracts, ZIP code tabulation areas (ZCTAs), NTAs, and boroughs.

As an exploratory analysis, we describe patterns of exposure to high HIV prevalence neighbourhoods. Data regarding the prevalence of HIV infection among the general population of adults were obtained for each ZCTA (AIDSVu, 2018) ZCTAs were classified into quartiles by estimated HIV prevalence per 100,000 population. The first quartile included ZCTAs with estimated HIV prevalence between 161 and 660 per 100,000 population; the second quartile included ZCTAs with estimated HIV prevalence between 661 and 1,190 per 100,000 population; the third quartile included ZCTAs with estimated HIV prevalence between 1,191 and 1,907 per 100,000 population; and the fourth quartile included ZCTAs with estimated HIV prevalence between 1,098 and 7,7975 per 100,000 population. We then assessed the distribution of the participants' residential neighbourhoods across these quartiles as well as the proportion of all GPS data points recorded within ZCTAs in each of these quartiles.

\section{Analysis}

Descriptive statistics were calculated for all survey variables, as well as variables derived from the GPS data, including frequencies and percentages for categorical variables and mean (with standard deviation [SD]) and median (with interquartile range [IQR]) for continuous variables.

\section{Results}

\section{Sample demographics}

The average age was 32.2 years (SD: 4.3). Most (71.4\%) were non-White, with $42.9 \%$ reporting their race as Black or African American. Most participants reported being in a relationship with a main partner (57.1\%). Most (57.1\%) were considered to be living in stable housing. Half (50.0\%) did not work full time or part time. All participants completed high school and $28.6 \%$ completed a bachelor's degree or higher. Most $(85.7 \%)$ reported an estimated yearly income of less than $\$ 20,000$. Most $(85.7 \%)$ had some form of health insurance. About one-third (35.7\%) reported their HIV status as HIV-positive.

\section{Survey assessment of frequently visited neighbourhood contexts}

Varying levels of concordance between the residential neighbourhood and each of the non-residential contexts were reported by participants. Among those who reported currently working $(n=7), 57.1 \%$ considered the neighbourhood they worked in to be different from the neighbourhood they lived in. Among those who considered their work neighbourhood to be different $(n=4), 75.0 \%$ worked in a different borough and all worked in a different NTA. Almost two-thirds (64.3\%) considered the neighbourhood that they socialised in most often in the past three months to be different from the neighbourhood they lived in. Among those who considered their social neighbourhood to be different ( $n=9$ ), $28.6 \%$ socialised in a different borough and $64.3 \%$ socialised in a different NTA. Among those who reported accessing healthcare services in the past three months $(n=13), 76.9 \%$ considered the neighbourhood that they received healthcare in most often in the past three months to be different from the neighbourhood they lived in. Among those who considered their healthcare neighbourhood to be different $(\mathrm{n}=10), 50.0 \%$ received healthcare in a different borough and $70.0 \%$ received healthcare in a different NTA.

\section{Global positioning system assessment of mobility}

A total of 641,242 points were recorded by the GPS devices, representing approximately 74.2 days' worth of mobility data. The mean number of points per participant was 49,657.2 (SD: 22,103.4) ( 5.7 days). Activity spaces created using a 200-meter buffer ranged from 4.4 square kilometres to 49.5 square kilometres, with a mean of 17.1 square kilometres (SD: 11.1).

While participants residential addresses represented 3 boroughs, 11 NTAs, 10 ZCTAs, and 12 census tracts, GPS data points were recorded in 4 of 5 boroughs $(80.0 \%), 124$ of 263 ZCTAs (47.1\%), and 709 of 2,168 census tracts $(32.7 \%)$. All participants had GPS data points recorded in two or more boroughs. The number of unique NTAs with GPS data points per participant ranged from 11 to 67, with a median of 19 NTAs (IQR: 9.0). The number of unique ZCTAs with GPS data points per participant ranged from 9 to 63, with a median of 19 ZCTAs (IQR: 5.8). The number of unique census tracts with GPS data points ranged from 35 to 235 , with a median of 71.5 census tracts (IQR: 32.8 ).

A map of ZCTAs by estimated HIV prevalence is displayed in Figure 1. Among participants with valid addresses $(n=12)$, twothirds $(66.7 \%)$ resided in ZCTAs with an estimated HIV prevalence higher than 1,908 per 100,000 population $(1.9 \%$ or higher, the highest quartile). Overall, $16.4 \%(n=105,055)$ of these data points were recorded in ZCTAs in the first (lowest) quartile of HIV prevalence estimates; $0.9 \%(n=5,504)$ were recorded in ZCTAs in the second quartile of HIV prevalence estimates; $24.5 \%$ $(n=157,421)$ were recorded in ZCTAs in the third quartile of HIV prevalence estimates; and $58.2 \%(n=373,262)$ were recorded in ZCTAs in the fourth (highest) quartile of HIV prevalence estimates. Participants' daily path areas intersected with an average of 23.3 ZCTAs (SD: 14.1), including an average of 12.5 ZCTAs (SD: 4.7 ) in the highest quartile of HIV prevalence estimates. The average area-weighted HIV prevalence estimate for participants' daily path areas was $2,788.2$ per 100,000 population (2.8\%) (SD: 592.0 ), ranging from a low of 1723.1 per 100,000 population $(1.7 \%)$ to a high of $3,704.6$ per 100,000 population $(3.7 \%)$.

\section{Discussion}

Spatial mobility patterns were examined through the use of mobility surveys and GPS data among a sample of TGW in NYC. We found that participants' activity spaces covered large areas over the span of a week and that participants visited diverse spatial contexts in the course of their daily activities. Among this sample, despite only residing in 12 census tracts, GPS data points were recorded in 709 unique census tracts. It was observed that most participants resided in and that most GPS data points were record- 
ed within ZCTAs within the highest quartile of estimated HIV prevalence in the general population (between 1,908 and 7,795 per 100,000 population). Although these findings cannot be used to explain the high estimated HIV prevalence among TGW (Baral et al., 2013), these findings suggest that the participants in this sample of TGW regularly frequent neighbourhoods with some of the highest estimates of HIV prevalence in NYC. Despite the small sample size, the association between place, community HIV prevalence, mobility, and factors that increase the vulnerability of TGW to HIV infection are worthy of future investigation in reducing the burden of the HIV epidemic in these communities.

This study extends previous research on spatial mobility, although we are not aware of any other studies that have been conducted on the role of place in the health and lives of gender minority individuals among other samples of transgender and gender non-conforming people. Although prior assessments of the concordance between frequently visited neighbourhood contexts (e.g., residential, work/school, social) have been survey-based (Duncan et al., 2014a; Perchoux et al., 2014), this study uses GPS technologies to directly assess the spatial contexts that individuals engage with and interact in, making this study highly innovative. The use of GPS technologies directly addresses the uncertain geographic context problem (Kwan, 2012) that arises from spatial uncertainty in the actual areas that exert contextual influences on the health and behaviours of individuals being studied and temporal uncertainty in the timing and duration in which individuals experience these contexts by identifying personal exposure areas with data on individual mobility patterns. This method, when implemented among larger samples of transgender and gender non-conforming individuals, may improve the accuracy of estimates of neighbourhood-level exposures.

Previous research has suggested that diverse populations experience multiple spatial contexts in the span of their daily lives, including other populations in NYC. For example, a recent surveybased assessment of concordance between commonly visited neighbourhood types among a sample of young MSM in NYC found that $75 \%$ of participants in the sample resided, socialised, and had sex in three different boroughs (Duncan et al., 2014b, 2016a); none have been conducted with transgender and gender non-conforming populations. Although the widespread discrimination faced by TGW in various public settings (Grant et al., 2011) was hypothesised to force spatial entrapment in this population, our study shows evidence for high levels of mobility across neighbourhood contexts. This may be due to the availability and accessibility of multiple modes of public transportation in NYC (Goetzke, 2008).

Future research should continue to examine spatial mobility to improve our understanding of the multiple types of neighbourhoods that influence health. Qualitative methods, such as semistructured interviews, and the integration of mobility surveys into maps derived from participant GPS data may provide insights into why participants choose to visit certain locations over others, including those within and outside the residential neighbourhood (Duncan et al., 2016b). This qualitative research can inform quantitative longitudinal studies to understand the pathways through which multiple, varied neighbourhood environments influence health, including among TGW. Future research can also assess socio-demographic differences in spatial polygamy, as mobility patterns may reflect the diversity of experiences within transgender communities by race/ethnicity and socioeconomic status (Reisner et al., 2014).
These findings are not without limitation. First, it is possible that participants could have changed their mobility patterns while wearing the GPS device, introducing potential reactivity bias and selective daily mobility bias. However, previous research has suggested that these issues will be minimal (Duncan et al., 2014b, 2016a; Goedel et al., 2017). Because the GPS protocol was implemented in a large metropolitan location, there are several known issues with the quality of GPS signals in areas with large buildings due to multipath reflectance (Suh and Shibasaki, 2007). In addition to this issue, individuals in NYC often travel via the underground subway system, where the GPS units are unable to obtain a signal from orbiting satellites, leading to additional data loss. Third, given that these participants only wore the GPS unit for a period of one week, it is unknown whether the spatial patterns observed in this study are representative of typical travel patterns. Collection of GPS data for longer periods of time and over time may improve the representativeness of the GPS data collected. In addition, this study was conducted among a small convenience sample of TGW in NYC. Several pilot studies assessing the acceptability and feasibility of GPS monitors to assess mobility and exposure to neighbourhood environments have had small samples, ranging from 10 to 20 participants (Royster et al., 2002; Mitchell et al., 2014; Byrnes et al., 2015; Macklin et al., 2018). Although the findings from this study may not be generalisable to TGW in other areas due to small sample size, further research is needed in larger samples, given the potential impact of inequities faced by transgender women on their mobility and access to resources.

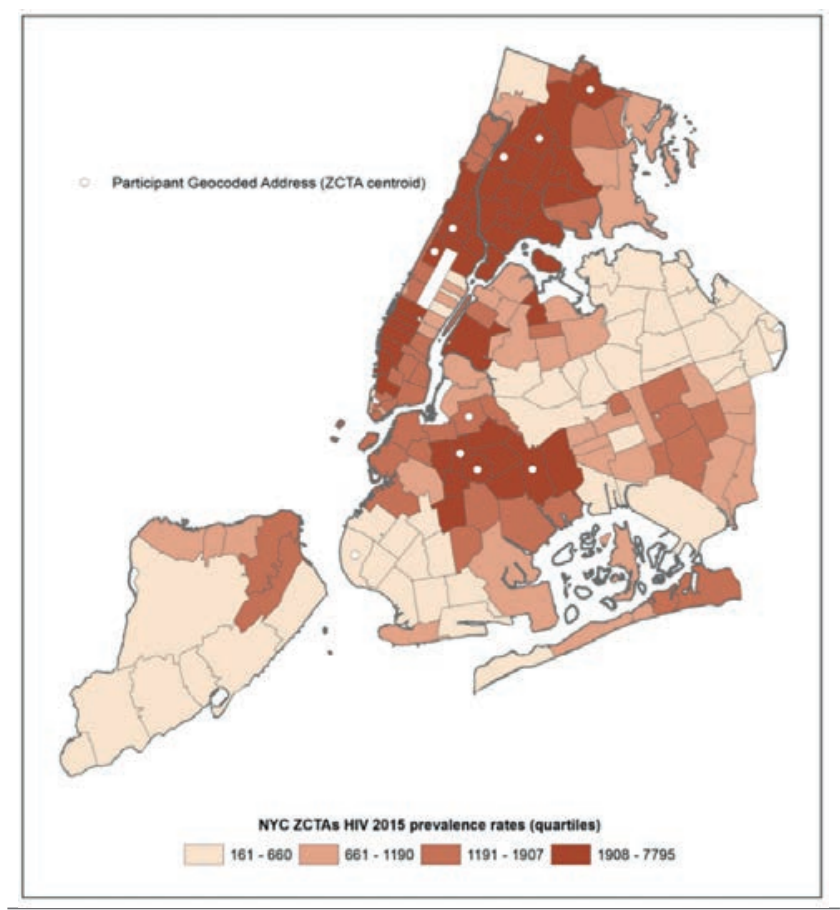

Figure 1. Quartiles of estimated HIV prevalence among residents at the zone improvement plan code tabulation area (ZCTA) level, New York City, 2015. 


\section{Conclusions}

Significant spatial mobility was observed in both survey and GPS-based assessments among this sample of TGW in NYC, suggesting that spatial entrapment may not be a significant factor in determining mobility in this population. In addition, more than half of all GPS points recorded were within neighbourhoods with the highest quartile of estimated HIV prevalence in the general population. Future studies of neighbourhood contexts and health among TGW should account for contexts beyond the residential neighbourhood to understand the role of neighbourhood exposures (e.g., community HIV prevalence) in patterns of health and disease in this population.

\section{References}

AIDSVu, 2018. AIDSVu. Available from: www.aidsvu.org

Baral SD, Poteat T, Strömdahl S, Wirtz AL, Guadamuz TE, Beyrer C, 2013. Worldwide burden of HIV in transgender women: a systematic review and meta-analysis. Lancet Infect Dis 13:214-22.

Byrnes HF, Miller BA, Wiebe DJ, Morrison CN, Remer LG, Wiehe SE, 2015. Tracking adolescents with global positioning system-enable cell phones to study contextual exposures and alcohol and marijuana use: a pilot study. J Adolesc Health 57:45-247.

Chaix B, 2009. Geographic life environments and coronary heart disease: a literature review, theoretical contributions, methodological updates, and a research agenda. Annu Rev Public Health 30:81-105.

Chaix B, Meline J, Duncan S, Merrien C, Karusisi N, Perchoux C, Lewin A, Labadi K, Kestens Y, 2013. GPS tracking in neighbourhood and health studies: a step forward for environmental exposure assessment, a step backward for causal inference? Health Place 21:46-51.

Cruz TM, 2014. Assessing access to care for transgender and gender nonconforming people: a consideration of diversity in combating discrimination. Soc Sci Med 110:65-73.

Duncan DT, Kapadia F, Halkitis PN, 2014. Examination of spatial polygamy among young gay, bisexual, and other men who have sex with men in New York City: the P18 cohort study. Int J Environ Res Public Health 11:8962-83.

Duncan DT, Kapadia F, Regan SD, Goedel WC, Levy MD, Barton SC, Friedman SR, Halkitis PN, 2016. Feasibility and acceptability of global positioning system (GPS) methods to study the spatial contexts of substance use and sexual risk behaviours among young men who have sex with men in New York City: a P18 cohort sub-study. PloS One 11:e0147520.

Duncan DT, Kawachi I, 2018. Neighbourhoods \& health. 2nd ed. Oxford University Press, New York, USA.

Duncan DT, Kawachi I, Subramanian S, Aldstadt J, Melly SJ, Williams DR, 2013. Examination of how neighbourhood definition influences measurements of youths' access to tobacco retailers: a methodological note on spatial misclassification. Am J Epidemiol 179:373-81.

Duncan DT, Méline J, Kestens Y, Day K, Elbel B, Trasande L, Chaix B, 2016. Walk score, transportation mode choice, and walking among French adults: a GPS, accelerometer, and mobility survey study. Int J Environ Res Public Health 13:611.

Duncan DT, Regan S, Chaix B, 2018. Operationalizing neighbor- hood definitions in health research: spatial misclassification and other issues. In: Duncan D, Kawachi I, eds. Neighbourhoods \& health. 2nd ed. Oxford University Press, New York, USA, pp 19-56.

Duncan DT, Regan SD, Shelley D, Day K, Ruff RR, Al-Bayan M, Elbel B, 2014. Application of global positioning system methods for the study of obesity and hypertension risk among lowincome housing residents in New York City: a spatial feasibility study. Geospat Health 9:57-70.

Duncan DT, Tamura K, Regan SD, Elbel B, Meline J, Al-Ajlouni YA, Chaix B, 2017. Quantifying spatial misclassification in exposure to noise complaints among low-income housing residents across New York City neighbourhoods: a global positioning system (GPS) study. Ann Epidemiol 27:67-75.

Edelman EA, 2011. "This area has been declared a prostitution free zone": discursive formations of space, the state, and trans "sex worker" bodies. J Homosex 58:848-64.

England KV, 1993. Suburban pink collar ghettos: the spatial entrapment of women? Ann Assoc Am Geogr 83:225-42.

Goedel WC, Reisner SL, Janssen AC, Poteat TC, Regan SD, Kreski NT, Confident G, Duncan DT, 2017. Acceptability and feasibility of using a novel geospatial method to measure neighbourhood contexts and mobility among transgender women in New York City. Transgend Health 2:96-106.

Goetzke F, 2008. Network effects in public transit use: evidence from a spatially autoregressive mode choice model for New York. Urban Stud 45:407-17.

Golledge R, Stimson R, 1997. Spatial cognition, cognitive mapping, and cognitive maps. In: Golledge R, Stimson R, eds. Spatial behavior: a geographic perspective. The Guilford Press, New York, USA, pp 224-266.

Grant JM, Mottet LA, Tanis J, Harrison J, Herman JL, Keisling M, 2011. Injustice at every turn: a report of the national transgender discrimination survey. Retrieved from Washington: https://www.transequality.org/sites/default/files/docs/resource s/NTDS_Report.pdf

Institute of Medicine, 2011. The health of lesbian, gay, bisexual, and transgender people: building a foundation for better understanding. Retrieved from Washington: https://www.nap. edu/catalog/13128/the-health-of-lesbian-gay-bisexual-andtransgender-people-building

Kwan MP, 1999. Gender and individual access to urban opportunities: a study using space-time measures. Prof Geogr 51:21027.

Kwan MP, 2012. The uncertain geographic context problem. Ann Assoc Am Geogr 102:958-68.

Macklin G, Stanton MC, Tchuem-Tchuenté LA, Stothard JR, 2018. A pilot study using wearable global positioning system data loggers to compare water contact levels: Schistosoma haematobium infection in pre-school-age children (PSAC) and their mothers at Barombi Kotto, Cameroon. Trans R Soc Trop Med Hyg 112:361-5.

Matthews SA, 2011. Spatial polygamy and the heterogeneity of place: studying people and place via egocentric methods. In: Burton LM, Kemp SP, Chui LM, Matthews SA, Takeuchi DT, eds. Communities, neighbourhoods, and health. Springer, New York, pp 35-55.

Mayer KH, Bradford JB, Makadon HJ, Stall R, Goldhammer H, Landers S, 2008. Sexual and gender minority health: what we know and what needs to be done. Am J Public Health 98:98995. 
Mitchell JT, Schick RS, Hallyburton M, Dennis MF, Kollins SH, Beckham JC, McClernon FJ, 2014. Combined ecological momentary assessment and global positioning system tracking to assess smoking behavior: a proof of concept study. J Dual Diagn 10:19-29.

Næss P, 2006. Urban structure matters: residential location, car dependence and travel behaviour. 1st ed. Routledge, Philadelphia.

Perchoux C, Chaix B, Cummins S, Kestens Y, 2013. Conceptualization and measurement of environmental exposure in epidemiology: accounting for activity space related to daily mobility. Health Place 21:86-93.

Perchoux C, Kestens Y, Thomas F, Van Hulst A, Thierry B, Chaix B, 2014. Assessing patterns of spatial behavior in health studies: their socio-demographic determinants and associations with transportation modes (the RECORD Cohort Study). Soc Sci Med 119:64-73.

Reisner SL, Bailey Z, Sevelius J, 2014. Racial/ethnic disparities in history of incarceration, experiences of victimization, and associated health indicators among transgender women in the US. Women Health 54:750-67.

Reisner SL, Hughto JMW, Dunham EE, Heflin KJ, Begenyi JBG, CoffeyEsquivel J, Cahill S, 2015. Legal protections in public accommodations settings: a critical public health issue for transgender and gender $\square$ nonconforming people. Milbank Q 93:484-515.

Royster MO, Hilborn ED, Barr D, Carty CL, Rhoney S, Walsh D, 2002. A pilot study of global positioning system and geographical information system measurement of residential proximity to agricultural fields and urinary organophosphate metabolite concentrations in toddlers. J Expo Anal Environ Epidemiol 12:433-40.

Spicer SS, Schwartz A, Barber ME, 2010. Special issue on homelessness and the transgender homeless population. J Gay Lesbian Mental Health 14:267-70.

Suh Y, Shibasaki R, 2007. Evaluation of satellite-based navigation services in complex urban environments using a three-dimensional GIS. IEICE T Commun 90:1816-25. 